\title{
Isobaric analog states of neutron-rich nuclei. Doppler shift as a measurement tool for resonance excitation functions
}

\author{
P. Boutachkov ${ }^{1, a}$, G.V. Rogachev ${ }^{1}$, V.Z. Goldberg ${ }^{2}$, A. Aprahamian ${ }^{1}$, F.D. Becchetti ${ }^{3}$, J.P. Bychowski ${ }^{4}$, Y. Chen ${ }^{3}$, \\ G. Chubarian ${ }^{2}$, P.A. DeYoung ${ }^{4}$, J.J. Kolata ${ }^{1}$, L.O. Lamm ${ }^{1}$, G.F. Peaslee ${ }^{4}$, M. Quinn ${ }^{1}$, B.B. Skorodumov ${ }^{1}$, and \\ A. Wohr ${ }^{1}$ \\ 1 Physics Department, University of Notre Dame, Notre Dame, IN 46556, USA \\ 2 Texas A\&M University, College Station, TX 77843, USA \\ 3 Physics Department, University of Michigan, Ann Arbor, MI 48109, USA \\ 4 Physics Department, Hope College, Holland, MI 49422, USA
}

Received: 1 October 2004 /

Published online: 21 April 2005 - (c) Società Italiana di Fisica / Springer-Verlag 2005

\begin{abstract}
We present a new approach for the measurement of resonance excitation functions of neutronrich nuclei using Doppler shift information. Preliminary data from the first application of the method is presented in the spectroscopy studies of ${ }^{7} \mathrm{He}$ isobaric analog states in ${ }^{7} \mathrm{Li}$.
\end{abstract}

PACS. 25.60.-t Reactions induced by unstable nuclei $-25.40 . \mathrm{Kv}$ Charge-exchange reactions $-27.20 .+\mathrm{n}$ $6 \leq A \leq 19$

\section{Introduction}

Radioactive beams provide new opportunities for spectroscopy studies of drip line nuclei. Neutron-rich beams can populate high isospin states which are isobaric analogs of even more exotic neutron-rich systems [1]. We propose a method in which high isospin states are populated in resonance interaction of protons with neutron-rich beams. A $\gamma$-ray is then detected from the daughter nucleus created in a subsequent neutron decay. Information about the total and differential cross-sections of the created high isospin states can be extracted from the shape of the observed Doppler shifted $\gamma$-spectrum.

\section{The method}

The thick target inverse geometry technique [2] was successfully used to study proton-rich nuclei $[3,4]$. Recently it has been used in studies of exotic neutron-rich systems $[5,6]$. In the latter experiments, the differential crosssection for $(p, p)$ and $(p, n)$ reactions populating high isospin states was measured at backward angles. The method presented here is a further development of the idea to study exotic neutron-rich nuclei through their isobaric analog states populated in well understood simple reactions [1]. We describe the technique using spectroscopy of ${ }^{7} \mathrm{He}$ isobaric analog states in ${ }^{7} \mathrm{Li}$ as an example.

In ${ }^{6} \mathrm{He}+p$ scattering, one can populate states with isospin $T=3 / 2$ in ${ }^{7} \mathrm{Li}$. These states are isobaric analogs of

\footnotetext{
${ }^{\text {a }}$ Conference presenter; e-mail: pboutach@nd.edu
}

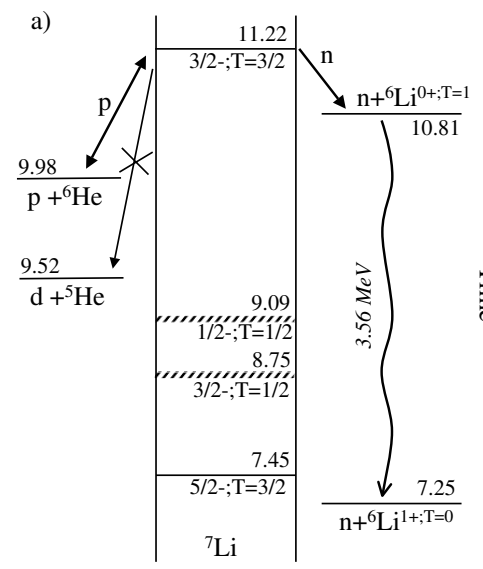

b)

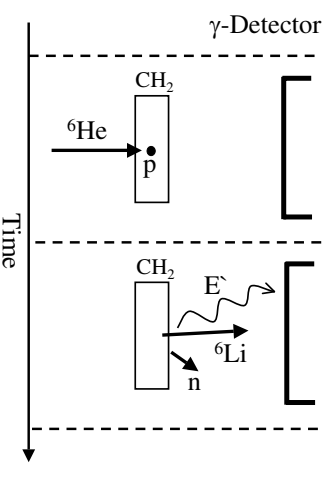

Fig. 1. a) Decay scheme for $T=3 / 2$ resonances in ${ }^{7} \mathrm{Li}$ and b) kinematic scheme of the experiment.

the levels in ${ }^{7} \mathrm{He}$. The populated $T=3 / 2$ states have only two open isospin allowed channels: proton decay back to ${ }^{6} \mathrm{He}$ or neutron decay to $T=1$ states of ${ }^{6} \mathrm{Li}$, see fig. $1 \mathrm{a}$. As follows from the wave function of the populated $T=3 / 2$ states,

$$
\frac{1}{\sqrt{3}} \Psi\left({ }^{6} \mathrm{He}\right) \Psi(p)+\sqrt{\frac{2}{3}} \Psi\left({ }^{6} \mathrm{Li}, T=1\right) \Psi(n),
$$

the neutron decay is dominant and the reduced decay widths for the open channels are related as $\gamma_{n} / \gamma_{p}=\sqrt{2}$. The $T=1$ state of ${ }^{6} \mathrm{Li}$ populated via the $(p, n)$ reaction can decay only by isospin forbidden channels. Therefore, 
the probability for $\gamma$ transition is enhanced with respect to the particle decays. For the specific case of the first $T=1$ state in ${ }^{6} \mathrm{Li}$, particle decay also violates the parity conservation law and therefore only $\gamma$ decay is allowed. Thus, measuring the characteristic $3.56 \mathrm{MeV} \gamma$-ray from the decay of the first $T=1$ state in ${ }^{6} \mathrm{Li}$ is a clear signature that a $T=3 / 2$ state in ${ }^{7} \mathrm{Li}$ was populated. This chain of transmutations is typical for the isobaric analog states of neutron-rich nuclei close to the line of stability. What is special for the described example is the $100 \%$ probability for $\gamma$ decay of the first $T=1$ state in ${ }^{6} \mathrm{Li}$.

Suppose that a thick proton target $\left(\left(\mathrm{CH}_{2}\right)_{n}\right)$ is used to stop the ${ }^{6} \mathrm{He}$ beam. Interaction of ${ }^{6} \mathrm{He}$ with protons can take place at any energy from the maximum (beam energy) to zero populating $T=1 / 2$ and $T=3 / 2$ resonances in ${ }^{7} \mathrm{Li}$. When a $T=3 / 2$ resonance is populated, it will decay with highest probability to neutron and ${ }^{6} \mathrm{Li}\left(0^{+}\right.$, $T=1$ ) state (see fig. 1). Velocity of ${ }^{6} \mathrm{Li}$ will depend on the velocity of ${ }^{7} \mathrm{Li}$ and the angle at which the neutron was emitted. The excited ${ }^{6} \mathrm{Li}$ nucleus decays by $\gamma$ emission before it loses any energy in the target (the width of $0^{+}, T=1$ resonance is $8 \mathrm{eV}$ ) and information on the velocity of ${ }^{6} \mathrm{Li}$ is preserved in the Doppler shift of the $\gamma$-ray. Therefore a $\gamma$-detector placed at a fixed angle will observe a Doppler shifted and broadened peak. The shift comes mainly from the velocity of ${ }^{7} \mathrm{Li}$ while the broadening will depend on the angular distribution of the emitted neutron. The magnitude of the peak will depend on the reaction yield. Therefore by using a detector at fixed angle with known absolute efficiency, one can extract information about the total and the differential cross-sections as a function of energy and angle in one run without changing the experimental conditions. In addition, the measurement is insensitive to the energy resolution of the beam.

\section{Isobaric analog states of ${ }^{7} \mathrm{He}$}

The method described above was first applied to the study of ${ }^{7} \mathrm{He}$ isobaric analog states. Figure 2 shows part of the spectrum from HPGe Clover detector placed at $0^{\circ}$ with respect to the beam velocity. The continuous curve corresponds to the population of the isobaric analog of the ${ }^{7} \mathrm{He}$ ground state in ${ }^{7} \mathrm{Li}$. The curve was obtained by folding the cross-section from a two channel $R$-matrix calculation and all kinematic effects, see sect. 2. There is no arbitrary normalization in the above calculation. The resonance parameters used for the g.s. were taken from ref. [6]. The contribution of the direct charge-exchange process was estimated with the code TWAVE [7] and was found to be negligible. Based on this calculation, one can see that the first peak in the spectrum is related to the g.s. and that there is a clear excess of counts at higher $\gamma$-ray energies which corresponds to ${ }^{6} \mathrm{Li}\left(0^{+}, T=1\right)$ nuclei with higher velocity (higher excitation energies of ${ }^{7} \mathrm{Li}$ ).

In the spectroscopic studs of ${ }^{7} \mathrm{He}$ an interesting finding was made by M. Meister et al. [8]. Evidence was obtained for a very low-lying $1 / 2^{-}$state (spin-orbit partner of the ground state) with essentially single-particle $\left({ }^{6} \mathrm{He}(\right.$ g.s. $\left.)+n\right)$ structure. An attempt to find the analog

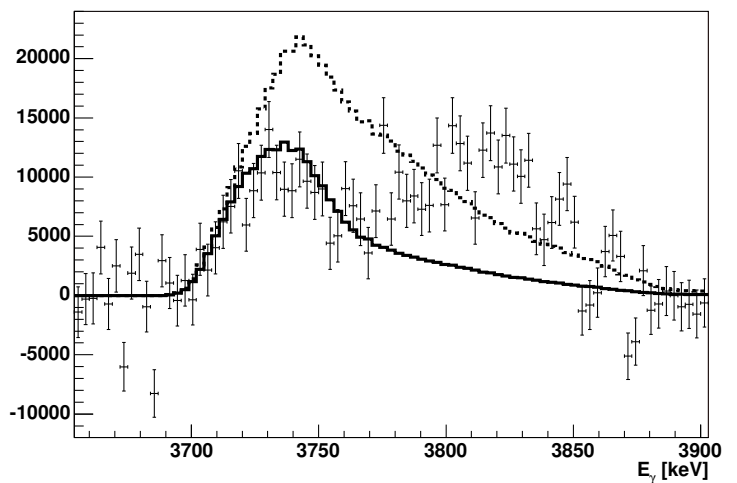

Fig. 2. Final spectrum of the Doppler shifted $3.56 \mathrm{MeV} \gamma$-rays obtained by subtraction of the carbon contribution form the $\mathrm{CH}_{2}$ target, a linear Compton background and dividing by the absolute detector efficiency. Solid line shows the contribution of the known g.s. resonance $T=3 / 2, J=3 / 2^{-}$in ${ }^{7} \mathrm{Li}$. Dotted line was obtained by taking into account the narrow low-lying $1 / 2^{-}$state proposed in [8].

of this resonance in ${ }^{7} \mathrm{Li}\left(T=3 / 21 / 2^{-}\right)$revealed no narrow resonances in this region [6]. The results obtained in ref. [6] are confirmed by the data presented here. The isobaric analog of a low-lying narrow $1 / 2^{-}$resonance in ${ }^{7} \mathrm{He}$ is not observed. The expected shape of the $\gamma$ spectrum in case of population of a resonance with parameters from ref. [8] is shown with a dotted line in fig. 2. It is clearly seen from the figure the magnitude and the shape of the data is not reproduced. The present results and these of [6] are completely independent since different techniques were used to measure different quantities. Therefore, the existence of the state with parameters proposed in [8] can be reliably excluded.

\section{Conclusion}

We propose a new method for spectroscopic studies of neutron-rich nuclei close to the border of stability. As an example, the ${ }^{7} \mathrm{He}$ isobaric analog states of ${ }^{7} \mathrm{Li}$ were studied. The measured $\gamma$-ray spectra show clear evidence that isobaric analog states of ${ }^{7} \mathrm{He}$ were excited. The existence of a narrow low-lying $1 / 2^{-}$state in ${ }^{7} \mathrm{He}$ is ruled out. We present evidence for higher-lying resonances in ${ }^{7} \mathrm{He}$. We believe that the presented technique will be very useful in the future for studies of nuclei at the drip line.

\section{References}

1. V.Z. Goldberg, in ENAM98: Exotic Nuclei and Atomic Masses, AIP Conf. Proc. 455, 319 (1998).

2. K.P. Artemov et al., Sov. J. Nucl. Phys. 52, 408 (1990).

3. L. Axelsson et al., Phys. Rev. C 54, R1511 (1996).

4. V.Z. Goldberg et al., JETP Lett. 67, 1013 (1998).

5. G.V. Rogachev et al., Phys. Rev. C 67, 041603 (2003).

6. G.V. Rogachev et al., Phys. Rev. Lett. 92, 232502 (2004).

7. S. Barua, private communication.

8. M. Meister et al., Phys. Rev. Lett. 88, 102501 (2002). 